
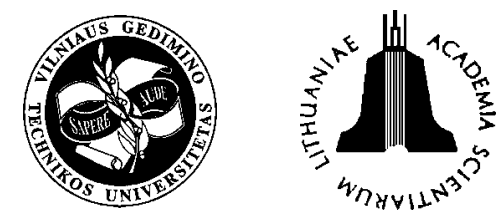

JOURNAL OF CIVIL ENGINEERING AND MANAGEMENT

http:/www.vtu.lt/english/editions

2004, Vol X, No 1, 3-14

\title{
DISCRETE ELEMENT METHOD AND ITS APPLICATION TO THE ANALYSIS OF PENETRATION INTO GRANULAR MEDIA
}

\author{
Robertas Balevičius ${ }^{1}$, Algis Džiugys ${ }^{2}$, Rimantas Kačianauskas ${ }^{3}$ \\ Laboratory of Numerical Modelling of Vilnius Gediminas Technical University, Saulètekio al. 11, \\ LT-10223Vilnius-40,Lithuania.E-mail:11Robertas.Balevicius@st.vtu.lt; ${ }^{2}$ dziugys@isag.lei.lt; 3 rkac@fm.vtu.lt
}

Received 6 May 2003; accepted 4 Nov 2003

\begin{abstract}
Application of discrete element method (DEM) to keel penetration in granular media is investigated. The basic relations for visco-elastic granular media composed of spherical particles are presented, together with 5th order Gear predictor-corrector scheme for time-integration. The background version of DEM and numerical time integration algorithm are developed and implemented into DEMMAT code. The implementation of time-integration algorithm is verified by simple tests concerning particle-particle, particle-wall interactions, for which analytical expressions exist. By limiting the size of the media domain, the three-dimensional problem is reduced to particular case presented as twodimensional domain of spherical particles. The variation of keel reaction and distribution of the particle forces due to different material properties are investigated.
\end{abstract}

Keywords: discrete element method, penetration, visco-elastic granular media.

\section{Introduction}

The response of granular media depends on the interaction between individual particles and their quantitative observation and registration are required [1]. The experimental measurements are extraordinarily difficult because the duration of interaction of the particles is very short and the displacements of individual particles are relatively small [2]. On the other hand, the increasing capacity of the advanced computer technologies opens up new vistas for modelling of the granular media by the discrete concept, which is an attractive alternative to continuum model [3].

Among various numerical simulation techniques, the discrete element method (DEM), initiated by P. Cundall and O. Strack [4], is one of the most powerful tools. The DEM allows for the simulation of particle motion and interaction between the particles, taking into account not only the obvious geometric and material effects such as particle shape, material non-linearity, viscosity, friction, etc, but also the effects of various physical fields of surrounding media, even of chemical reactions [5]. Recently, the DEM has been used for the solution of discrete and continuous problems including solid, fluid and molecular mechanics, heat transfer, etc [2, 5-10].

One of the most promising areas of future applications of the discrete element method seems to be geotechnical engineering. The discrete approach assumes the soil as an assembly of granules or discrete particles, where macromechanical behaviour of soil is predefined by micromechanical intergranular properties.
Large varieties of engineering applications are related to dynamic actions of different machine parts and mechanical tools contacting the soil. A penetration problem belongs to the category of relatively simple but fundamental problems of geotechnical engineering [3, 1113]. Its qualitative and quantitative description forms the basis for future investigations in terms of micromechanical models as well as providing suitable software tools.

It should be noted that commercial codes of the DEM have not been developed to such an extent to be applied for a wide variety of problems. As a rule, their main disadvantage lies, for example [14], in the fact that they do not allow to handle new specific models. For the above reasons, the development of specific software tools and codes is open for future investigations remaining promising activity.

The current work presents the application of DEM technique to the analysis of penetration in granular media. The background version of DEM and the numerical time-integration algorithm are developed and implemented into the original DEMMAT code. The quality of implementation is verified by physically observable behaviour of interactions of particle-particle, particle-wall and by the comparison of results of the present tests with the analytical solutions.

The developed DEMMAT code was applied to simulation of keel penetration into visco-elastic granular media composed of spherical particles. By limiting the size of the media domain, the three-dimensional problem may be reduced to a particular case presented as two-dimen- 
sional domain of spherical particles. The variation of keel reaction and distribution of particle forces due to different material properties of granular media are investigated.

\section{Discrete state formulation}

The granular media present a space filled by the particles termed here as discrete elements. The media are assumed to be composed of spherical particles with different radii $R_{i}$. The particles are assumed to be deformable bodies, deforming each other by normal and shear forces.

The composition of media is time-dependent because individual particles change their position by free rigid body motion or by contacting with neighbour particles or walls. Each particle may be in contact with other particles.

The visco-elastic material of granular media is defined by the modulus of elasticity, Poisson's ratio and damping coefficients in normal and shear directions.

The boundary conditions of media are determined by planes and treated as particles with an infinite radius and mass. The external action is induced with kinematic boundary conditions which are implemented by the walls movements.

The dynamic behaviour of media is considered as the dynamics of each particle. Consequently, the overall response of media is predicted by the behaviour of individual particles, the dynamics of which is evaluated by applying the second Newton's law. One of the most important issues considered by a discrete approach is the detection of interaction forces between contacting particles. The interaction forces of each contacting pair are locally resolved on the basis of actual geometry and the appropriate constitutive relationships employed. This chapter focuses mainly on the description of geometry of kinematic contact between two spherical particles, inter-particle contact forces and boundary conditions.

\subsection{Geometry of kinematic contact of spherical particles}

The kinematics and contact geometry of spherical particles are considered by normal component acting in the normal direction denoted by subscript $n$ and the tangential component denoted by subscript $t$. Consequently, contact deformation of the particle $i$ in respect of another particle $j$ may influence the contact kinetics of particle $i$ in respect of particle $k$.

Two spherical particles in the contact, $i$ and $j$, are defined by the positions $x_{i}$ and $x_{j}$ of the centres of gravity $O_{i}$ and $O_{j}$ of the particles and by the velocities $v_{i}$ and $\boldsymbol{v}_{i}$, and the rotation velocities $\boldsymbol{w}_{i}$ and $\boldsymbol{w}_{j}$ (Fig 1). Relations presented below generally reflect the DEM model with rotation previously developed by Džiugys [10, 15].

The vector $\boldsymbol{x}_{i j}$ of the relative position points from the centre of gravity of particle $i$ to that of particle $j$

$$
\mathbf{x}_{i j}=\mathbf{x}_{i}-\mathbf{x}_{j}
$$

The contact point $C_{i j}$ is defined to be in the centre of the overlap area with the position vector $\mathbf{x}_{c i j}$. The depth of the overlap is defined by $h_{i}$

$$
h_{i j}=R_{i}+R_{j}-\left|\mathbf{x}_{i j}\right|,
$$

where $R_{i}$ and $R_{j}$ are the radii of the spherical particles $i$ and $j$.

The normal vector $\mathbf{n}_{i j}$ is a unit vector pointing in the direction of the contact surface through the centre of the overlap area towards the particle $i$

$$
\mathbf{n}_{i j}=\frac{\mathbf{x}_{i j}}{\left|\mathbf{x}_{i j}\right|} .
$$

It holds that

$$
\mathbf{n}_{i j}=-\mathbf{n}_{j i} .
$$

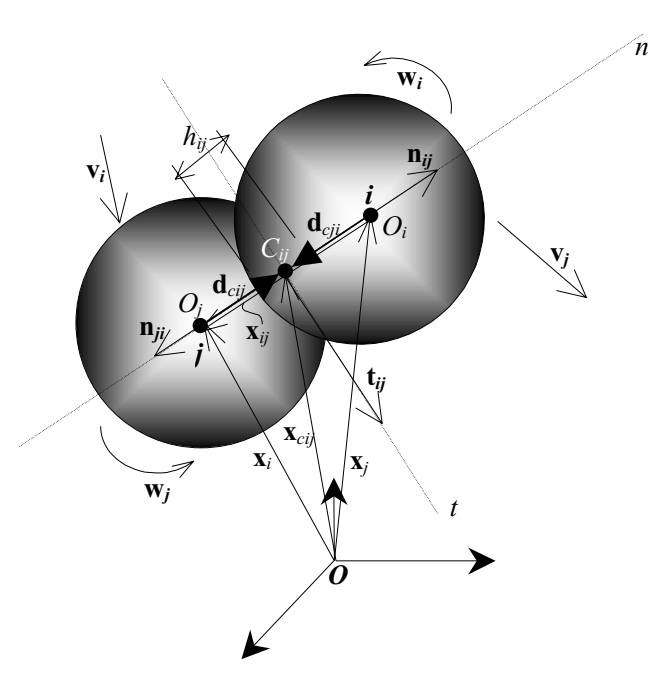

Fig 1. Geometry of overlapping contact of two particles

The vectors $\mathbf{d}_{c i j}$ and $\mathbf{d}_{c j i}$ are pointing in the direction from the centre of the particles to the contact point $C_{i j}$

$$
\mathbf{d}_{c i j}=\mathbf{x}_{c i j}-\mathbf{x}_{i}=-\left(R_{i}-\frac{1}{2} h_{i j}\right) \mathbf{n}_{i j} .
$$

The relative velocity of the contact point (Fig 2) is defined as:

$$
\mathbf{v}_{i j}=\mathbf{v}_{c i j}-\mathbf{v}_{c j i}
$$

where

$$
\begin{gathered}
\mathbf{v}_{c i j}=\mathbf{v}_{i}+\mathbf{w}_{i} \times \mathbf{d}_{c i j}, \\
\mathbf{v}_{c j i}=\mathbf{v}_{j}+\mathbf{w}_{j} \times \mathbf{d}_{c j i},
\end{gathered}
$$

are the velocities of the particles $i$ and $j$ at the contact point. It holds that

$$
\mathbf{v}_{i j}=-\mathbf{v}_{j i} .
$$

The angle between vectors of relative velocity $\mathbf{v}_{i j}$ and unit vector $\mathbf{n}_{i j}$ can be defined by the expression 


$$
\cos \varphi=\frac{\mathbf{v}_{i j} \cdot \mathbf{n}_{i j}}{\left|\mathbf{v}_{i j}\right| \cdot\left|\mathbf{n}_{i j}\right|} .
$$

The vector of an angle between global and local coordinates system is expressed by

$$
\alpha=\arccos \left(n_{i j}\right) \text {. }
$$

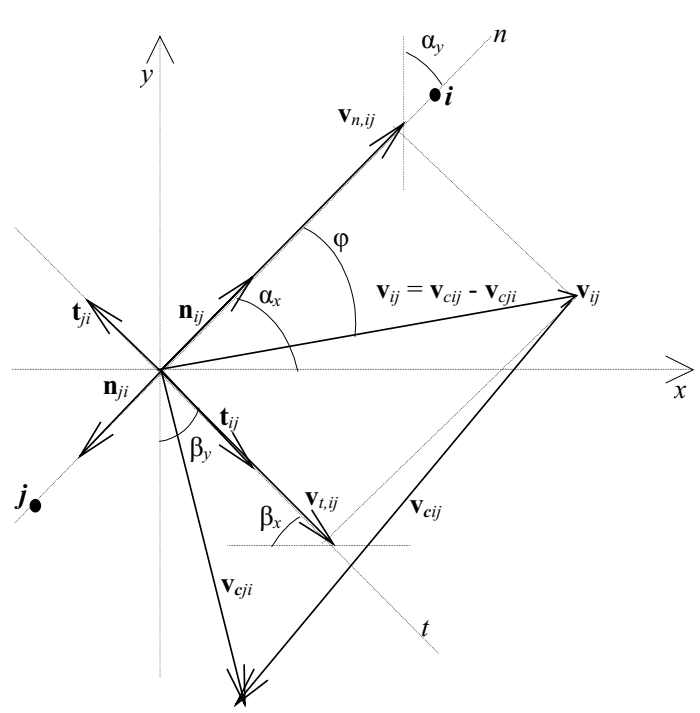

Fig 2. A scheme of the relative velocity vectors

Taking into account relations (10-11) the modulus of normal contact relative velocities $\mathbf{v}_{n, i j}$ is obtained as

$$
\left|\mathbf{v}_{n, i j}\right|=\left|\mathbf{v}_{i j}\right| \cdot \cos \varphi=\frac{\mathbf{v}_{i j} \cdot \mathbf{n}_{i j}}{\left|\mathbf{n}_{i j}\right|} .
$$

Taking into account relations (10-12) the normal component of contact relative velocities $\mathbf{v}_{n, i j}$ is obtained in the following way:

$$
\begin{gathered}
\mathbf{v}_{n, i j}=\left|\mathbf{v}_{n, i j}\right| \alpha=\left|\mathbf{v}_{n, i j}\right| \mathbf{n}_{i j}, \\
\mathbf{v}_{n, i j}=\left(\mathbf{v}_{i j} \cdot \mathbf{n}_{i j}\right) \mathbf{n}_{i j} .
\end{gathered}
$$

The tangential component of the relative velocity of the contact point may be established by vector summation

$$
\mathbf{v}_{t, i j}=\mathbf{v}_{i j}-\mathbf{v}_{n, i j} .
$$

The slip distance follows from temporal integration of the relative velocity, starting at the point in time when particles $i$ and $j$ come into contact. The slip distance is expressed by the vector of tangential displacement $\boldsymbol{\delta}_{t, i j}$ and is defined by the expressions given in $[1,10]$

$$
\delta_{t, i j}=\int \mathbf{v}_{t, i j}(t) d t .
$$

If the tangential component of the contact velocity $\mathbf{v}_{t, i j}$ is not equal to zero, then the unit vector $\mathbf{t}_{i j}$ of the tangential contact direction is directed along $\mathbf{v}_{t, i j}$. If $\mathbf{v}_{t, i j}$ is equal to zero, $\mathbf{t}_{i j}$ has the same direction in which the slip occurs. Otherwise $\mathbf{t}_{i j}$ is equal to zero if $\mathbf{v}_{t, i j}$ and $\boldsymbol{\delta}_{t, i j}$ are equal to zero. Finally,

$$
\mathbf{t}_{i j}=\cos \beta= \begin{cases}\frac{\mathbf{v}_{t, i j}}{\left|\mathbf{v}_{t, i j}\right|}, & \mathbf{v}_{t, i j} \neq 0 \\ \frac{\delta_{t, i j}}{\left|\delta_{t, i j}\right|}, & \mathbf{v}_{t, i j}=0, \quad \delta_{t, i j} \neq 0 \\ 0, & \text { otherwise }\end{cases}
$$

\subsection{Inter-particle contact forces}

A model of inter-particle visco-elastic contact forces describes the following effects: particle elasticity and energy dissipation in normal and tangential directions (Fig 3).

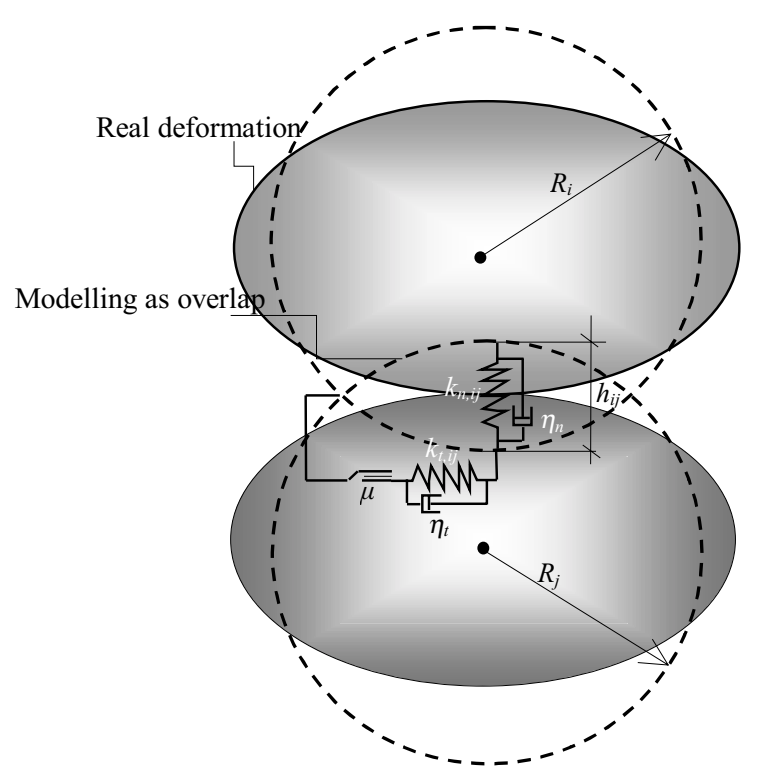

Fig 3. A model of inter-particle contact forces

The contact force $\mathbf{F}_{i j}$ of a visco-elastic collision between two particles $i$ and $j$ acts on the contact point $C_{i j}$ and can be expressed as sum of the normal and the tangential components

$$
\mathbf{F}_{i j}=\mathbf{F}_{n, i j}+\mathbf{F}_{t, i j} .
$$

A model for the normal repulsion force depends on the contact geometry and on the properties of the particle material $[10,16]$ :

$$
\begin{aligned}
& \mathbf{F}_{n, i j, \text { elastic }}=\frac{4}{3} \cdot \frac{E_{i} E_{j}}{E_{i}\left(1-v_{j}^{2}\right)+E_{j}\left(1-v_{i}^{2}\right)^{R i j}} h_{i j}^{\alpha-\alpha} \mathbf{n}_{i j}, \\
& \text { where } \\
& \qquad R_{i j}=\frac{R_{i} R_{j}}{R_{i}+R_{j}}
\end{aligned}
$$

is the reduced radius of two particles. 
For the Hertz contact model the power coefficient $\alpha=3 / 2$, while for Hook's law $\alpha=1 ; E_{i}$ and $E_{j}, v_{i}$ and $v_{j}$ - are elastic moduli and Poisson's ratios of particles $i$ and $j$.

In the normal direction, the energy dissipates during real collisions between the particles (Fig 3). The dissipation can be modelled by the non-conservative viscous force during collision. Based on the linear dependency of the force on the relative velocity of the particles at the contact point (14) with a constant normal damping coefficient $\gamma_{n}[10,17]$

$$
\mathbf{F}_{n, i j, v i s c o u s}=-\gamma_{n} m_{i j} \mathbf{v}_{n, i j} \text {, }
$$

where

$$
m_{i j}=\frac{m_{i} m_{j}}{m_{i}+m_{j}}
$$

is the reduced mass of the contacting particles $i$ and $j$.

The formulation of model for the tangential force is more difficult, since the phenomena of tangential deformation and friction (static and dynamic) have to be modelled, whereas, in general, they depend on the normal force $\mathbf{F}_{n, i j}$ and depth of overlap $h_{i j}$ as well as on the history of the tangential contact force. The model of energy dissipation in tangential direction must also be included for static friction; otherwise perpetual oscillations in the time of static friction will occur.

Two major approaches can be found in literature to model tangential forces: global models, where the tangential force is modelled by one law, and separated models, where static and dynamic friction are modeled by separate equations. The most popular approach comprises the evolution of tangential force $\mathbf{F}_{t, i j}$ divided into parts of static friction or dynamic friction

$$
\mathbf{F}_{t, i j}=-\mathbf{t}_{i j} \min \left(\left|\mathbf{F}_{t, i j, \text { static }} \| \mathbf{F}_{t, i j, \text { dynamic }}\right|\right) .
$$

Dynamic friction force can be described by the equation

$$
\mathbf{F}_{t, i j, \text { dynamic }}=-\mu\left|\mathbf{F}_{n, i j}\right| \mathbf{t}_{i j} .
$$

The expression (24) of the dynamic friction force (where $\mu$ is friction coefficient) is widely used, but there is no common agreement about the model for the static friction. The most popular approach is based on the assumption that static friction can be calculated by the sum of energy dissipation and spring models

$$
\mathbf{F}_{t, i j, \text { static }}=\mathbf{F}_{t, i j, \text { elastic }}+\mathbf{F}_{t, i j, \text { viscous }} .
$$

The most general form of the spring model for static friction was used by Kohring [18, 19] in simplified expressions of Mindlin's [19] theory, assuming that no partial slipping of the contact surface occurs

$$
\mathbf{F}_{t, i j, \text { elastic }}=-\frac{16}{3} \cdot \frac{G_{i} G_{j} \sqrt{R_{i j} h_{i j}}}{G_{i}\left(2-v_{j}\right)+G_{j}\left(2-v_{i}\right)} \delta_{t, i j} \mathbf{t}_{i j},
$$

where $G_{i}$ and $G_{j}$ are the shear moduli of particles $i$ and $j$.

The model for energy dissipation in tangential direction was adequate to the model for energy dissipation in the normal direction (21)

$$
\mathbf{F}_{t, i j, v i s c o u s}=-\gamma_{t} m_{i j} \mathbf{v}_{t, i j},
$$

where $\gamma_{t}$ is tangential damping coefficient.

\subsection{The surrounding media}

The effects of the surrounding media or physical fields acting on the particle may be added as additional forces. In the current approach the effect of gravity field is taken into account.

\subsection{The boundary conditions}

The setting up of boundary conditions is very important for correct simulation of the granular material behaviour. The walls constructed by planes may be treated as the particles with an infinite radius and mass (Fig 4).

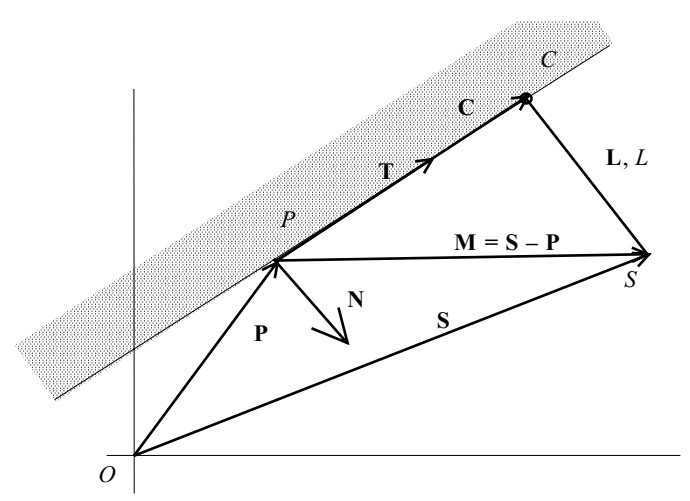

Fig 4. Geometry of boundary plane and neighbour point $S$

The geometry of boundary plane surface is defined by equation

$$
A x+B y+C z+D=0,
$$

where $(A, B, C) \neq(0,0,0)$ are the components of the plane surface normal vector $\mathbf{N}$.

The equation for every point $\mathbf{R}(x, y, z)$ of the boundary surface is defined as $(\mathbf{N} \cdot \mathbf{R})+D=0$. Therefore, if we have point $\mathbf{P}\left(x_{p}, y_{p}, z_{p}\right)$ of the plane, we can calculate constant $D$ by expression $D=-\left(A x_{p}+B y_{p}+C z_{p}\right)=-(\mathbf{N} \cdot \mathbf{P})$. If we have a point on the plane and a normal vector of the plane, it is easy to calculate the distance $\boldsymbol{L}$ from the plane to the adjacent point $S$. 


\section{A numerical algorithm}

\subsection{The basic relations}

The time-driven discrete element method was used to simulate the behaviour of granular media, which means that the mutual impact of particles is approximated by a representative overlap area or volume of particle shapes in the vicinity of the point of impact. The motion of each particle $i$ in time $t$ is described by the second Newton's law:

$$
\begin{gathered}
m_{i} \frac{d^{2} \mathbf{x}_{i}}{d t^{2}}=\mathbf{F}_{i}, \\
I_{i} \frac{d^{2} \theta_{i}}{d t^{2}}=\mathbf{T}_{i}
\end{gathered}
$$

where $\mathbf{x}_{i}, \theta_{i}$ are vectors of the position of the centre of gravity and orientation of the particle, $m_{i}$ is the mass of the particle $i(i=1, N), I_{i}$ is the inertia moment of the particle.

Vectors $\mathbf{F}_{i}$ and $\mathbf{T}_{i}$ present the sum of gravity and contact forces and torques, which act on the particle $i$ respectively. The contact forces is defined by relat

$$
\mathbf{F}_{i, \text { contact }}=\sum_{j=1, j \neq i}^{N} \mathbf{F}_{i j},
$$

where $\mathbf{F}_{i j}$ is a force acting on the contact area of elastic impacts between the particles $i$ and $j$.

The summation of torques $\mathbf{T}_{i \text {,contact }}$ is caused by the contact forces between particles

$$
\mathbf{T}_{i, \text { contact }}=\sum_{j=1, j \neq i}^{N} \mathbf{T}_{i j}=\sum_{j=1, j \neq i}^{N} \mathbf{d}_{c i j} \times \mathbf{F}_{i j} .
$$

The kinematic relations of equation (29-30) are expressed by (1-17) while the forces and torques are represented by (18-27).

\subsection{The time integration}

The state of all particles at the time $t$ is obtained by the integration of the equations (29-30). The numerical solution of these equations is obtained by a $5^{\text {th }}$-order Gear predictor-corrector [10, 20] scheme.

First, the particles positions $\mathbf{x}_{i}^{p}$, velocities $\mathbf{v}_{i}^{p}$, accelerations $\mathbf{a}_{i}^{p}$ and higher order dimensionless time derivatives $\mathbf{b}_{n i}^{p}=\frac{d^{n} \mathbf{x}}{d t^{n}} \frac{\Delta t^{n}}{n !}$ (where $\left.n=3,4,5\right)$, denoted hereafter by superscript $\mathrm{p}$, are predicted by expressions:

$$
\begin{aligned}
& \mathbf{x}_{i}^{p}(t+\Delta t)= \\
& \mathbf{x}_{i}(t)+\Delta t \mathbf{v}_{i}(t)+\frac{1}{2} \Delta t^{2} \mathbf{a}_{i}(t)+\mathbf{b}_{3 i}(t)+\mathbf{b}_{4 i}(t)+\mathbf{b}_{5 i}(t),
\end{aligned}
$$

$$
\mathbf{v}_{i}^{p}(t+\Delta t)=\mathbf{v}_{i}(t)+\Delta t \mathbf{a}_{i}(t)+\frac{1}{\Delta t}\left(3 \mathbf{b}_{3 i}(t)+4 \mathbf{b}_{4 i}(t)+5 \mathbf{b}_{5 i}(t)\right)
$$

$$
\begin{gathered}
\mathbf{a}_{i}^{p}(t+\Delta t)=\mathbf{a}_{i}(t)+\frac{2}{\Delta t^{2}}\left(3 \mathbf{b}_{3 i}(t)+6 \mathbf{b}_{4 i}(t)+10 \mathbf{b}_{5 i}(t)\right) \\
\mathbf{b}_{3 i}^{p}(t+\Delta t)=\mathbf{b}_{3 i}(t)+4 \mathbf{b}_{4 i}(t)+10 \mathbf{b}_{5 i}(t) \\
\mathbf{b}_{4 i}^{p}(t+\Delta t)=\mathbf{b}_{4 i}(t)+5 \mathbf{b}_{5 i}(t) \\
\mathbf{b}_{5 i}^{p}(t+\Delta t)=\mathbf{b}_{5 i}(t)
\end{gathered}
$$

After the prediction, the particle forces and accelerations are calculated according to the new positions and velocities taking into account relationship (29)

$$
\mathbf{a}_{i}^{c}(t+\Delta t)=\frac{\mathbf{F}_{i}\left(\mathbf{x}_{i}^{p}, \mathbf{v}_{i}^{p}\right)}{m_{i}} .
$$

Finally, the positions, velocities and higher-order time derivatives), denoted hereafter by superscript c, are corrected:

$$
\Delta \mathbf{a}_{i}(t+\Delta t)=\mathbf{a}_{i}^{c}(t+\Delta t)-\mathbf{a}_{i}^{p}(t+\Delta t),
$$

$$
\left(\begin{array}{c}
\mathbf{x}_{i}^{c}(t+\Delta t) \\
\mathbf{v}_{i}^{c}(t+\Delta t) \\
\mathbf{a}_{i}^{c}(t+\Delta t) \\
\mathbf{b}_{3 i}^{c}(t+\Delta t) \\
\mathbf{b}_{4 i}^{c}(t+\Delta t) \\
\mathbf{b}_{5 i}^{c}(t+\Delta t)
\end{array}\right)=\left(\begin{array}{l}
\mathbf{x}_{i}^{p}(t+\Delta t) \\
\mathbf{v}_{i}^{p}(t+\Delta t) \\
\mathbf{a}_{i}^{p}(t+\Delta t) \\
\mathbf{b}_{3 i}^{p}(t+\Delta t) \\
\mathbf{b}_{4 i}^{p}(t+\Delta t) \\
\mathbf{b}_{5 i}^{p}(t+\Delta t)
\end{array}\right)+\left(\begin{array}{l}
0.5 c_{0} \Delta t^{2} \\
0.5 c_{1} \Delta t \\
c_{2} \\
0.5 c_{3} \Delta t^{2} \\
0.5 c_{5} \Delta t^{2} \\
0.5 c_{5} \Delta t^{2}
\end{array}\right) \Delta \mathbf{a}_{i}(t+\Delta t) .(41)
$$

The values of the constants $c_{i}$ depend on the desired accuracy, and for a second order of differential equation are $c_{0}=3 / 16, c_{1}=251 / 360, c_{2}=1.0$, $c_{3}=11 / 18, c_{4}=1 / 6$ and $c_{5}=1 / 60$ [20].

The time step, $\mathrm{D} t$, of the integration of the particle position, velocity, orientation and angular velocity is a basic parameter of accurate results of simulations and depends on the time of contact. Two main criteria are applied to a time step:

1. Cundall and Strack [4] proposed that the time step must be smaller than the critical time step,

$$
\Delta t_{c}=\sqrt{\frac{m}{k}},
$$

which was estimated on the basis of the single degreeof-freedom system of the mass $m$ connected to the ground by a spring of stiffness $k$.

2. For sufficiently accurate simulations, the overlap depth must be considerably smaller than the radius of the particle $h_{\max }<<R_{i}$, therefore time step must be considerably smaller than the time of collision between two particles $\Delta t<<T_{c}$. 
In spite of the assertion of Thompson and Grest [21] that an accurate simulation requires $\Delta t \sim T_{c} / 50$, other authors use a larger time step. Dury and Ristow [22] used $\Delta t=T_{c} / 15$, Langston et al [23] $-\Delta t \sim T_{c} / 30$. Analysis of accuracy of numerical integration of equations (2930) using various integration schemes will be presented below.

\subsection{Computer implementation}

The application of DEM to a system involves the following basic steps:

1. Set-up of initial conditions of the particles and the walls.

2. Searching for the contacts between particles.

3. Applying interaction laws (calculating forces and moments) to all particle-particle and particle-wall contacts.

4. Applying Newton's second law to determine particle motion (29-30). Predicting positions, velocities, accelerations and higher-order time derivatives according to (33-38).

5. Updating the current state of particles and the walls according to (40-41).

6. Assigning a new time $t=t+\Delta t$.

7. If current time is within entire time period $(t \leq \mathrm{T})-$ go to the item 2, otherwise go to the next item.

8. Post processing, visualisation.

An original program called DEMMAT has been written in Compaq Visual FORTRAN language to implement the algorithm and method described above.

\section{Validation tests}

The validation tests were performed to check two aspects the algorithm and the developed software. Firstly, unidirectional contact motion test of a single particle was checked and the results were compared with analytical solutions. Secondly, the validity of the model was confirmed on the basis of the realistic physical observations.

The evaluation of the time integration scheme depends on calculation time resource presenting the most important part of the numerical simulation technique of DEM. Various integration schemes for the numerical solution of differential equations (29-30) can be used, but, according to Sundaram and Collins [24] at least the third-order accuracy is required to accurately track the particle trajectories. The Taylor Expansion Series and Velocity Verlet are the simplest and mostly used schemes. For granular materials the Taylor Expansion Series was used by Taguchi [25], and Velocity Verlet was applied by Akiyama [26], Kopf et al [27]. Recently, more sophisticated but more accurate $5^{\text {th }}$-order Gear predictorcorrector $[10,20]$ has come into its own rights. To obtain sufficiently accurate numerical simulations and adequate analytical solutions, the above three time integration schemes were used in current investigation.
During the first validation procedure the time integration schemes with different time steps were examined. Here, several models of normal contact forces were considered:

Test 1: normal elastic force;

Test 2: normal elastic and damping forces;

Test 3: normal elastic, damping and gravity forces.

To test the particle-wall collision, the particle moving at the initial velocity and hitting the wall's base were simulated. To test the particle-particle collision, an identical test is conducted, but the particle hits a stationary particle. In these tests, the tangential forces are set to zero. Test model data is described in Table 1.

Table 1. Test model data

\begin{tabular}{l|c|c}
\hline Quantity & Symbol & Value \\
\hline Particles radii, $\mathrm{m}$ & $R$ & 0,05 \\
\hline Particle mass, kg & $m$ & 10,0 \\
\hline Wall mass, kg & $m$ & $\infty$ \\
\hline Particle and wall elastic modulus, $\mathrm{Pa}$ & $E$ & $3 \cdot 10^{5}$ \\
\hline Particle and wall Poisson's ratio & $v$ & 0,30 \\
\hline Normal damping coefficient & $\gamma_{n}$ & 10,0 \\
\hline Initial velocity, m/s & $\mathrm{v}_{0}$ & 1,0 \\
\hline
\end{tabular}

In order to estimate the artificial damping of these time-integration schemes, the following relationship was applied

$$
\Delta_{x}=\frac{1}{N} \sqrt{\sum_{i=1}^{n}\left(x_{\text {analytical }}(t)-x_{\text {method }}(t)\right)^{2}},
$$

where $\Delta_{x}-$ artificial damping error, while $x_{\text {analytical }}(t)$ is a position of particle at time $t$ defined by analytical expressions $[28,29]$ according to the test models and $x_{\text {method }}(t)$ is a position of particle at time $t$ defined by time integration schemes according to the test models.

Table 2. Test model results

\begin{tabular}{l|c|c}
\hline \multirow{2}{*}{ Scheme } & \multicolumn{2}{|c}{ Artificial damping error, $\Delta_{x}$} \\
\cline { 2 - 3 } & $\Delta t=T_{c} / 10$ & $\Delta t=T_{c} / 30$ \\
\hline Test 1: & 0,000018 & 0,000002 \\
\hline $\begin{array}{l}5^{\text {th }}-\text { order Gear predictor- } \\
\text { corrector }\end{array}$ & 0,000179 & 0,000021 \\
\hline Velocity Verlet & 0,006101 & 0,002079 \\
\hline Taylor Expansion Series & 0,000111 & 0,000033 \\
\hline Test 2: & 0,000300 & 0,000082 \\
\hline $5^{\text {th }}$-order Gear predictor- \\
corrector \\
\hline Velocity Verlet & 0,000657 & 0,000139 \\
\hline Taylor Expansion Series & 0,000427 & 0,000034 \\
\hline Test 3: & 0,000594 & 0,000083 \\
\hline $\begin{array}{l}5^{\text {th }}-\text { order Gear predictor- } \\
\text { corrector }\end{array}$ & 0,000805 & 0,000140 \\
\hline Velocity Verlet & \\
\hline Taylor Expansion Series &
\end{tabular}


The results of adequacy tests of analytical and numerical solutions for different time integration schemes depending on time steps $\Delta t$ and the time of collision $T_{c}$ are presented in Table 2. As one can see, the accuracy mainly depends on dissipations and the number of active forces. The analysis of the artificial damping error shows that accurate simulation requires the ratio $\Delta t=T_{c} / 30$. The trajectory of particle motion is illustrated in Fig 5.
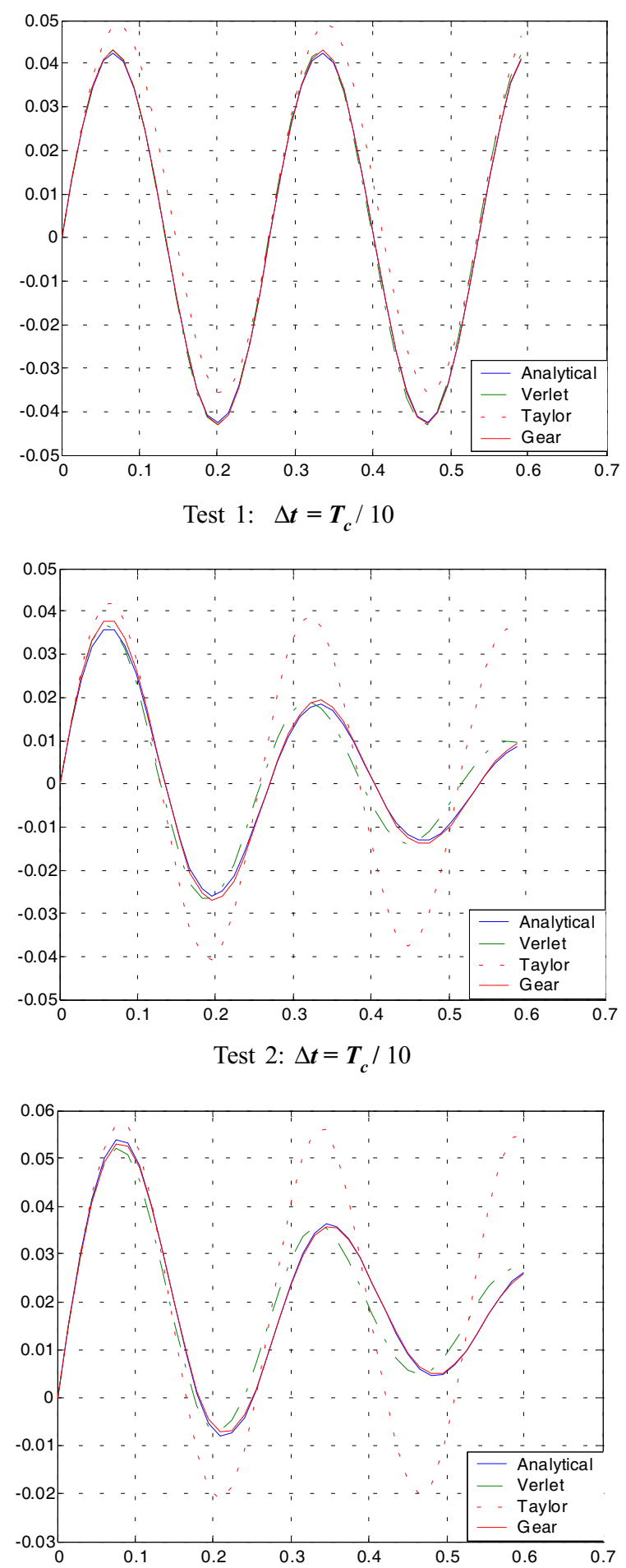

Test 3: $\Delta \boldsymbol{t}=\boldsymbol{T}_{\boldsymbol{c}} / 10$
According to Table 2, the $5^{\text {th }}$-order Gear predictor-corrector scheme results in the lowest artificial damping error. The Velocity Verlet algorithm resulted in slightly higher errors, whereas the Taylor Expansion Series expansion leads to considerably higher lowest artificial damping error. In order to obtain sufficiently accurate numerical simulations the $5^{\text {th }}$-order Gear predictor-corrector scheme was used in further development.

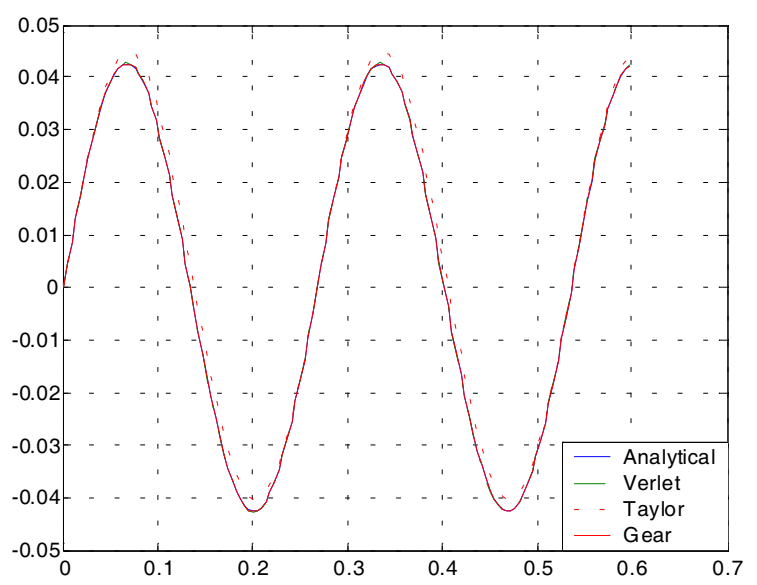

Test 1: $\Delta \boldsymbol{t}=\boldsymbol{T}_{\boldsymbol{c}} / 30$
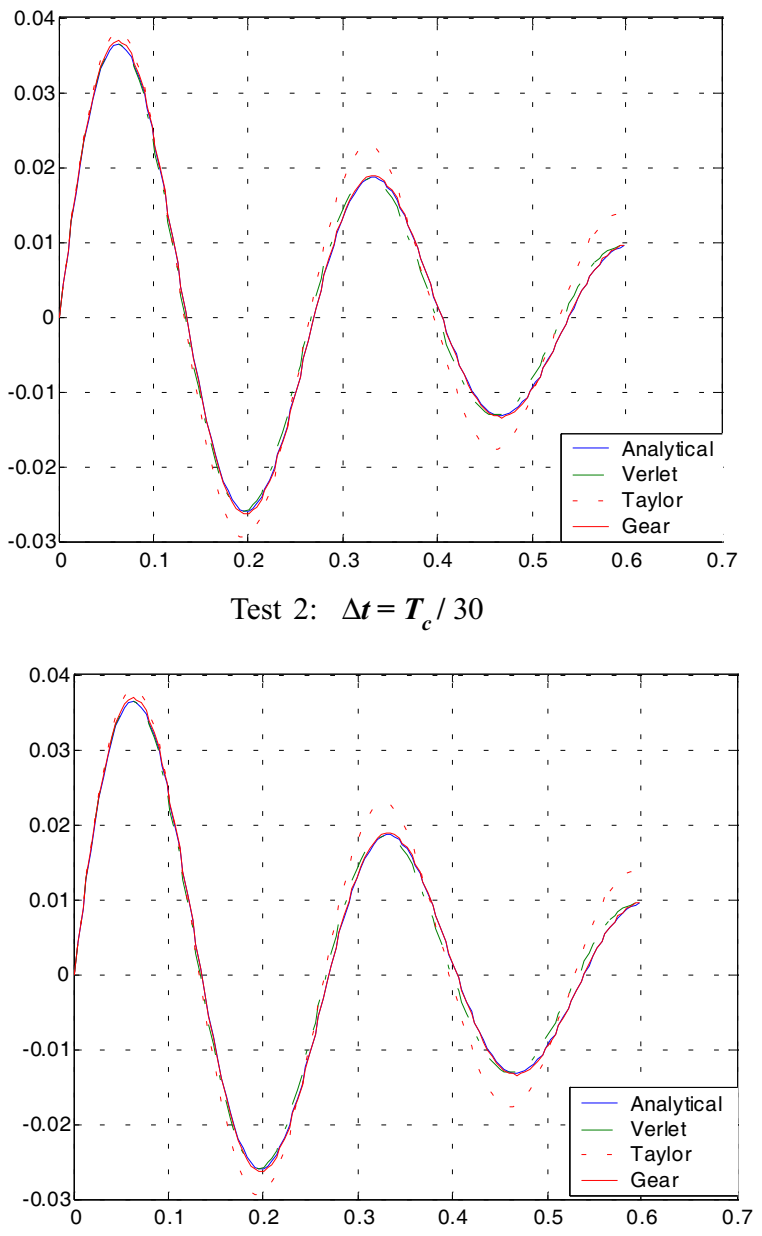

Test 3: $\Delta \boldsymbol{t}=\boldsymbol{T}_{\boldsymbol{c}} / 30$

Fig 5. The trajectory of particle motion for different time integration schemes versus the time step $\Delta t$ and the time of collision $\boldsymbol{T}_{\boldsymbol{c}}$ 
The validity of the above algorithm and the model was also checked in simulations of particle behaviour in the $3 \mathrm{D}$ wall's box in the real time. The initial data described in Table 1 are supplemented with the value of particle density $\rho=1000 \mathrm{~kg} / \mathrm{m}^{3}$, the friction coefficient $\mu=0,5$ as well as the normal and tangential damping coefficients $\gamma_{n}=10 \mathrm{~s}^{-1}, \gamma_{t}=5 \mathrm{~s}^{-1}$. The initial scheme and results of numerical simulation of particle motion under gravity force and initial velocity $v_{0 x}=0,5 \mathrm{~m} / \mathrm{s}$ are presented in Fig 6.

Fig $6 \mathrm{~b}$ shows the trajectory of an elastic particle which moves at the horizontal initial velocity under the force of gravity. Merely, the dynamic friction effect on the trajectory of particle is taken into account. As can be seen, the particle rebounds horizontally between the two walls of the box. The movement of the particle

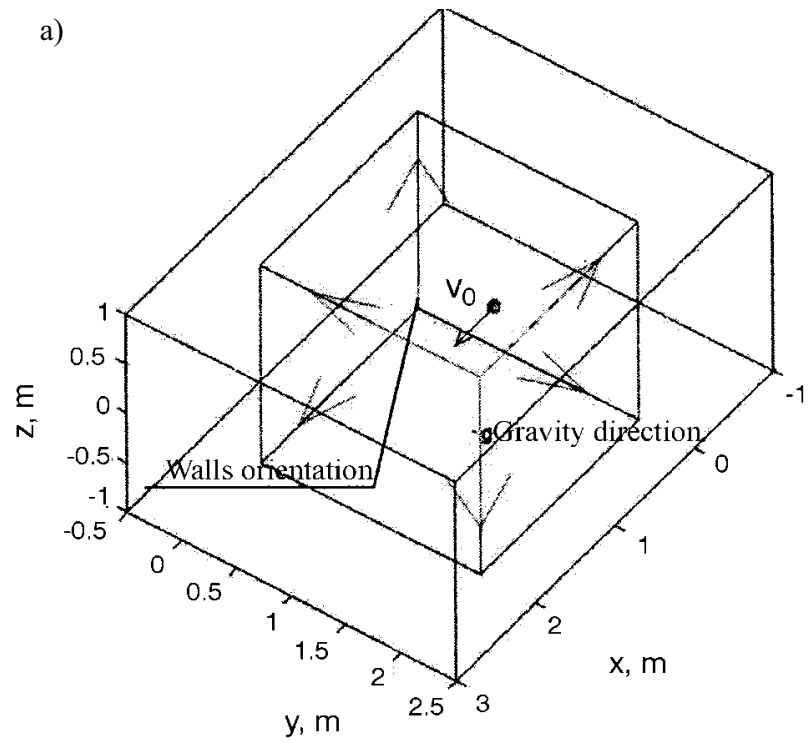

c)

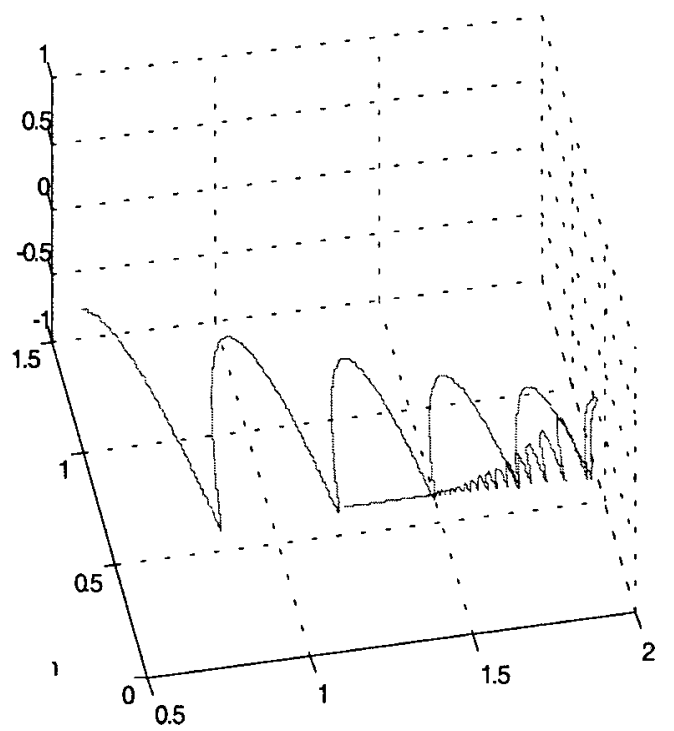

changes in the manner similar to a linear spring. The delay of particle motion may be explained by the fact, that a part of translation energy is transformed into rotational energy when the particle comes into contact with the wall.

Fig 6c shows the trajectory of a visco-elastic particle, where energy dissipation is caused by tangential and normal damping. When the particle rebounds, it fails to reach the original height and its height decays due to damping.

The trajectory of the elastic particle under action of the initial velocities $v_{0 x}=v_{0 y}=0,5 \mathrm{~m} / \mathrm{s}$ is presented in Fig 6d. Here, the energy dissipation is not taken into account and viscous forces (21) and (7) are set to zero. As can be seen, the particle rebounds with horizontal trajectory between the four walls of the box.

b)

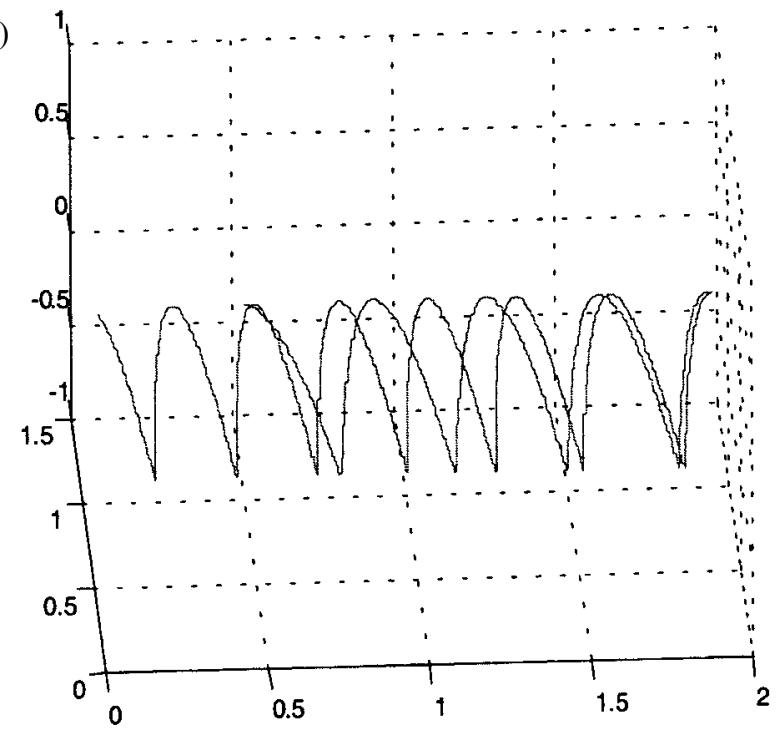

d)

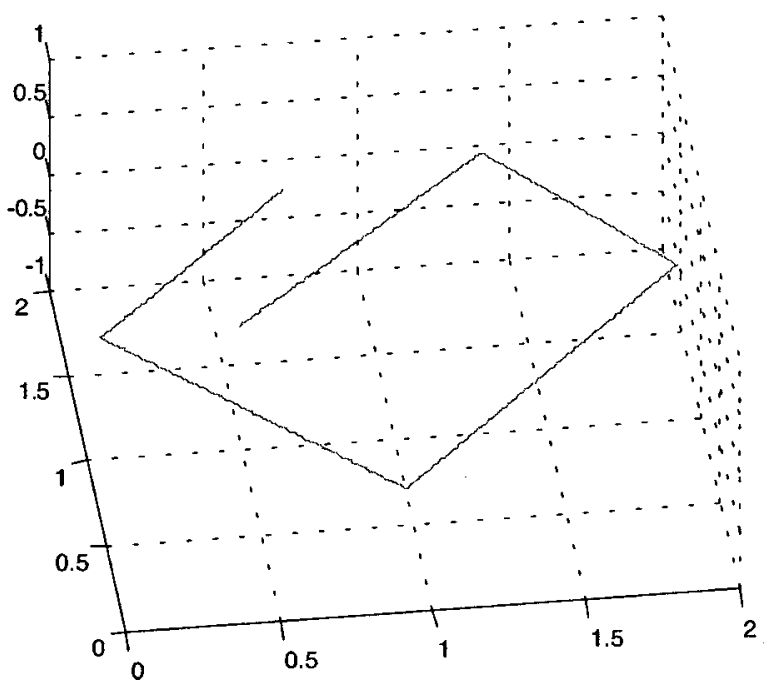

Fig 6. Simulation of particles trajectory in 3D box: a) initial scheme; b) graph of elastic particle trajectory with effect gravity and dynamic friction forces; c) the same of visco-elastic particle; d) graph of particle trajectory under action of the initial velocities $v_{0 x}, v_{0 y}$ 


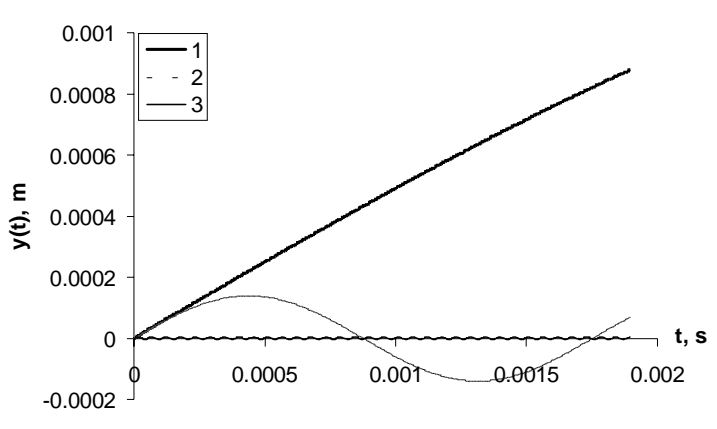

Fig 7. Trajectory of particle with different elasticity moduli after collision with the wall: $1-E=10^{6} \mathrm{MPa}, 2-E=10^{8}$ $\mathrm{MPa}, 3-E=10^{11} \mathrm{MPa}$

The following example illustrates the effect of elasticity properties. The particle with different elasticity moduli and constant velocity $v_{y}=0,5 \mathrm{~m} / \mathrm{s}$ hits the wall. The relatively low value $\left(\mathrm{E}=10^{6} \mathrm{~Pa}\right)$, the medium value $\left(\mathrm{E}=10^{8} \mathrm{~Pa}\right)$ and high value $\left(\mathrm{E}=10^{8} \mathrm{~Pa}\right)$ of elasticity moduli have been considered.

Fig 7 presents the trajectory of the particle after the contact with the wall. It shows the effect of spring elasticity on the amplitude and frequencies of oscillation of the particle. The high elasticity particle has a smaller amplitude and higher frequency of oscillation than a particle with a low modulus of elasticity. This effect is also discussed below in considering the behaviour of granular media with different elasticity properties.

\section{Penetration problem}

The granules are subjected to the action of the keel at the constant moving velocity $v_{k}$ and placed into an open box with deformable walls, the central section of which is shown in Fig 8.

The discrete approach described above generally presents a three-dimensional model. By reducing the thickness of the box until it reaches of two times of radius of particle $a=2 R_{\max }$, the problem, actually, is transformed into a two-dimensional one. This $2 \mathrm{D}$ model are

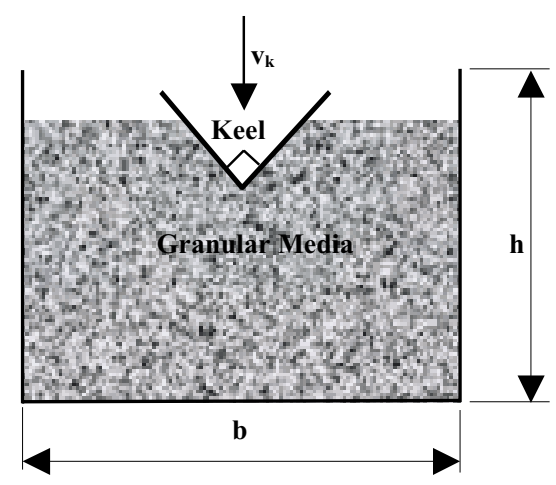

Fig 8. Illustration of penetration problem investigated and solved numerically. The 2D media contains about 2000 spherical particles.

The plane dimensions of the box under consideration are taken as $b=0,25 \mathrm{~m}$ and $h=0,15 \mathrm{~m}$. The deformed boundary is constructed by the particles of infinite radius. The initial data of the model are given in Table 3.

Table 3. Initial data of the penetration problem model

\begin{tabular}{l|c|l}
\hline Quantity & Symbol & Value \\
\hline Particle radii, $\mathrm{mm}$ & $R$ & $3-5$ \\
\hline Material density, $\mathrm{kg} / \mathrm{m}^{3}$ & $\rho$ & 2000 \\
\hline Poisson's ratio & $v$ & 0,20 \\
\hline Elastic moduli, $\mathrm{Pa}$ & $E$ & $10^{6}, 10^{8}, 10^{11}$ \\
\hline Shear modulus, $\mathrm{Pa}$ & $G$ & $0,3 \cdot 10^{6}$ \\
\hline $\begin{array}{l}\text { Normal damping coefficient, } \\
\text { 1/s }\end{array}$ & $\gamma_{n}$ & 10,0 \\
\hline Shear damping coefficient, $1 / \mathrm{s}$ & $\gamma_{t}$ & 10,0 \\
\hline Friction coefficient & $\mu$ & 0,3 \\
\hline Keel velocity, cm/s & $\mathrm{v}_{k}$ & 10 \\
\hline Time step, $\mathrm{s}$ & $\Delta t$ & $1 \cdot 10^{-5}, 1 \cdot 10^{-6}$, \\
& & $2 \cdot 10^{-7}$ \\
\hline Time simulation stopped, $\mathrm{s}$ & $T$ & 0,5 \\
\hline
\end{tabular}

The initial position of the particles was obtained by particles motion under gravity force while the particles reached the rest state and its kinetic energy was near to zero. The simulation of the penetration problem is carried out by introducing the keel, which is constructed of two walls and can be moved down at constant velocity $v_{k x}=0, v_{k y}=-0,1 \mathrm{~m} / \mathrm{s}$.

The numerical simulation is performed for the particles with different elastic properties. The relatively low $\left(E=10^{6} \mathrm{~Pa}\right)$, medium $\left(E=10^{8} \mathrm{~Pa}\right)$ and high $\left(E=10^{11}\right.$ $\mathrm{Pa})$ moduli of elasticity of particles are analysed.

The results of 2D simulation for different elasticity moduli are presented in Figs 9-11. The composition of particles illustrates the deformed shape of granular media while the column illustrates the particle force. The particles average force is shown in grey colour. The scale of blackness of gray colour was obtained by summing up of inter-particle contact forces

$$
f_{i}=\sum_{i \neq j}\left|\mathbf{F}_{i j}\right|
$$

As can be seen (Figs 9-11), the distributions of particle forces depend on the particle moduli of elasticity dramatically. The forces of granular media with low $\left(E=10^{6} \mathrm{~Pa}\right)$ elasticity modulus distribute more or less uniformly in each particle. The forces of granular media with high $\left(E=10^{11} \mathrm{~Pa}\right)$ elasticity moduli are not uniformly distributed. Forces acting upon most of the particles equal zero, but there are some particles acted by high forces.

Vertical $F_{k y}$ and horizontal $F_{k x}$ components of the reaction of the keel were obtained by summing up verti- 
a)

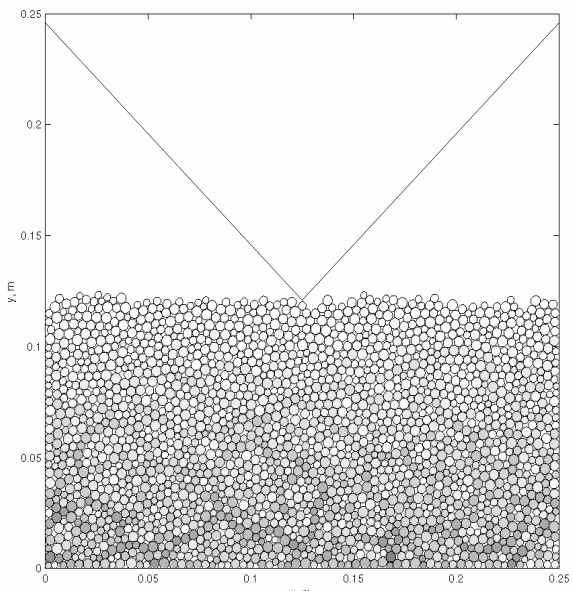

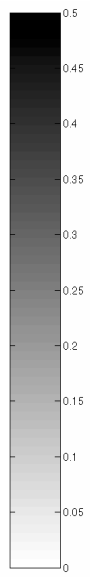

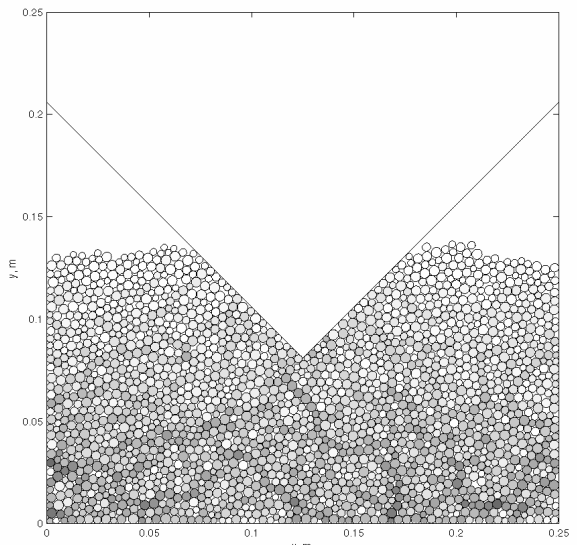

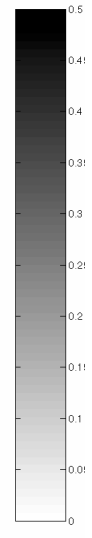

Fig 9. Simulation of penetration in granular media with a low $\left(E=10^{6} \mathrm{~Pa}\right)$ elasticity modulus a) initial distribution of forces; b) deformation of media and distribution of forces in $0,4 \mathrm{~s}$ (forces given in $\mathrm{N}$ )

a)

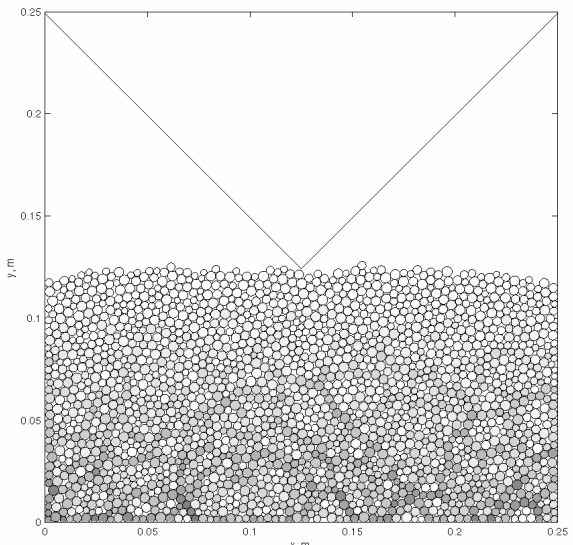

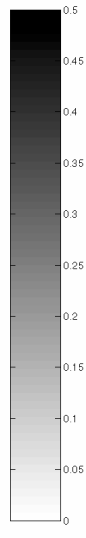

b)

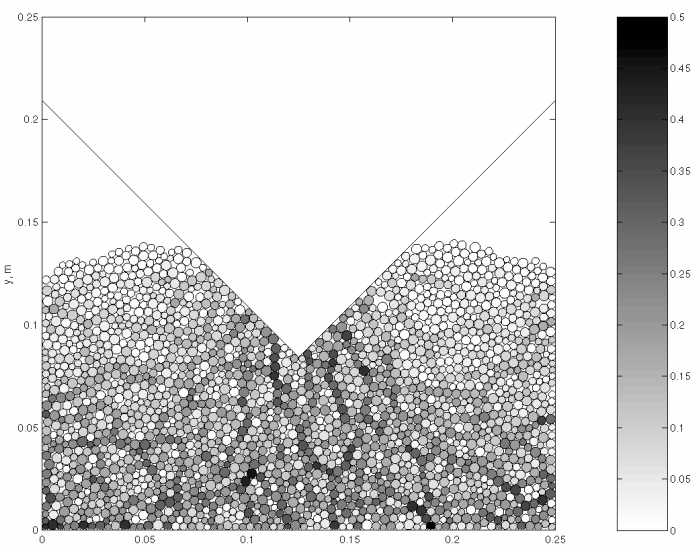

Fig 10. Simulation of penetration in granular media with a medium $\left(E=10^{8} \mathrm{~Pa}\right)$ elasticity modulus: a) initial distribution of forces; b) deformation of media and distribution of forces in $0,4 \mathrm{~s}$ (forces given in $\mathrm{N}$ )

a)

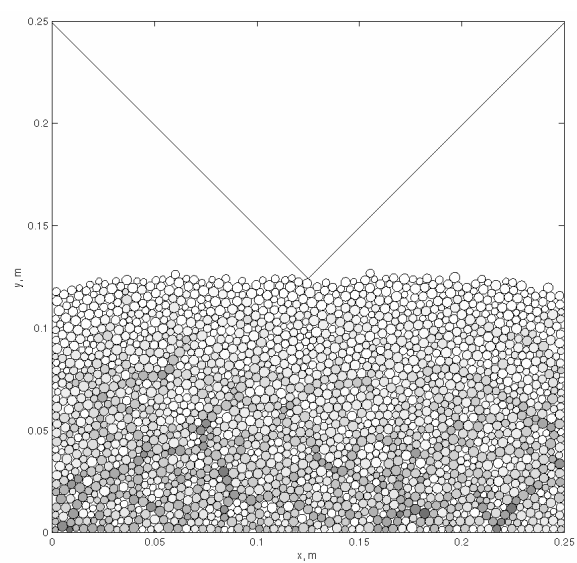

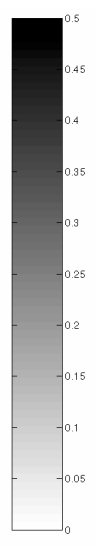

b)

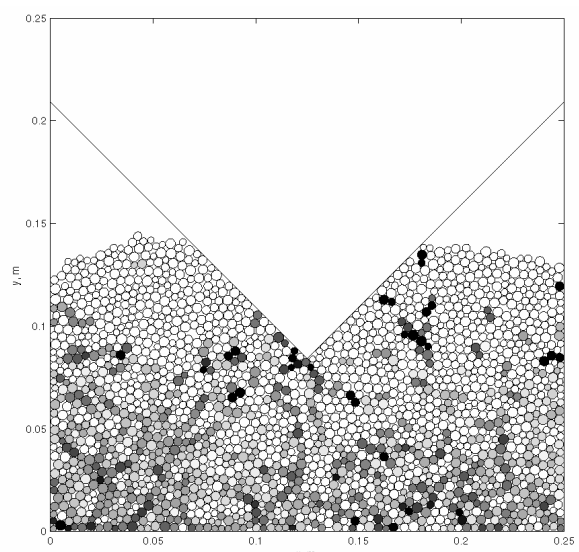

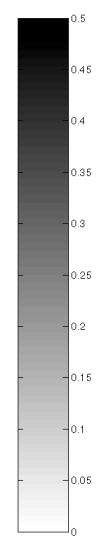

Fig 11. Simulation of penetration in granular media with a high $\left(E=10^{11} \mathrm{~Pa}\right)$ elasticity modulus: a) initial distribution of forces; b) deformation of media and distribution of forces in $0,4 \mathrm{~s}$ (forces given in $\mathrm{N}$ ) 
cal and horizontal components of the left and right side forces of the keel:

$$
\begin{aligned}
& F_{k y}=F_{r y}+F_{l y}, \\
& F_{k x}=F_{r x}+F_{l x},
\end{aligned}
$$

where $F_{r y}, F_{l y}-$ keel's right and left side vertical force component respectively, $F_{r x}, F_{l x}$ - the same for horizontal component.

In order to smoothen the pulsation of average reactions of the keel, $\tau$ time rectangular filter was applied

$$
\left\langle\mathrm{F}_{k}(t)\right\rangle=\frac{1}{\tau} \int_{t-\tau / 2}^{t+\tau / 2} \mathrm{~F}_{k}(\tau) d \tau,
$$

where $\tau$ - width of the filter.

The time variation of the absolute and relative vertical average reaction of the keel for different elasticity moduli are shown in Figs 12-13.

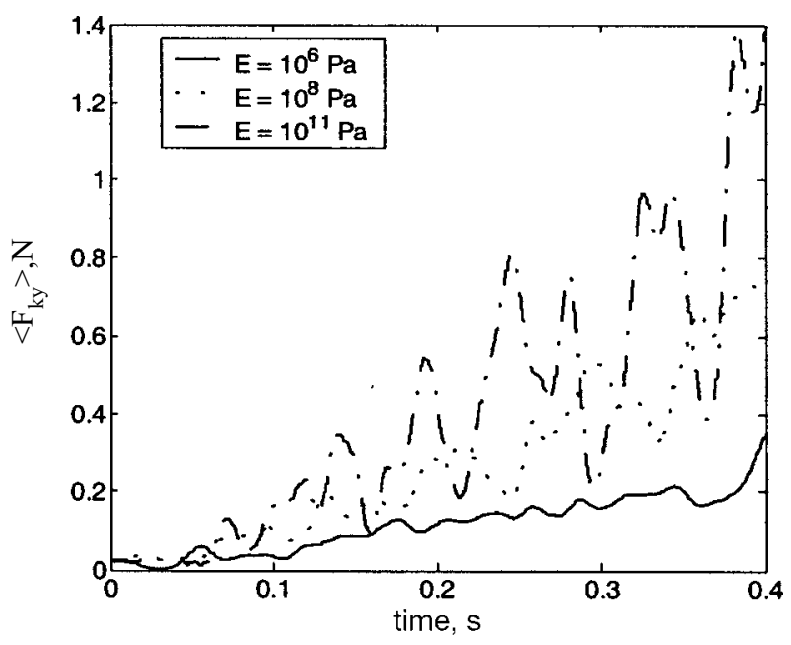

Fig 12. The evolution of the filtered vertical reaction force of the keel (rectangular filter width $\tau=0,01 \mathrm{~s}$ ) for three cases of the elastic moduli $E$ of particles

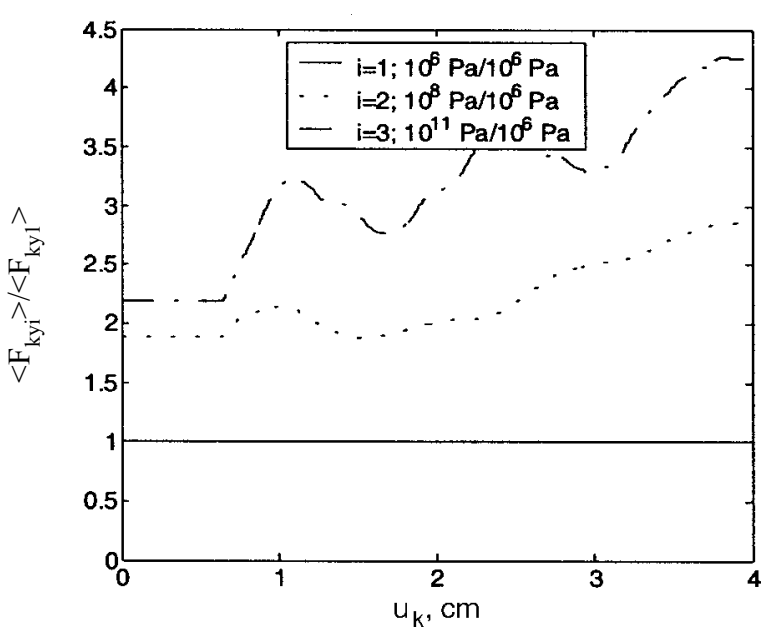

Fig 13. The dependence of the ratio of filtered vertical reaction force (rectangular filter width $\tau=0,0065 \mathrm{~s}$ ) on keel displacement
The analysis of numerical results (Figs 12-13) shows that curves of vertical average reaction of the keel are irregular due to the irregular local deformation of individual particles.

The values of the average horizontal driving force of the keel was about zero, but its oscillation amplitude increase with higher elasticity of the particles. In general, it was stated that the influence of particles size distribution on a horizontal driving force is very small.

\section{Conclusions}

The results obtained in the present investigation may be generally described as follows:

1. The described discrete element model composed of visco-elastic spherical particles is implemented into the developed DEMMAT code, the quality of which is proved by the solution of standard tests. This code open for new elements and interaction models may be considered as the first step in the development of an advanced simulation tool for granular and other inhomogeneous materials and is intended for modelling more complex geotechnical problems.

2. The time integration tests conducted with particle-particle and particle-wall interactions have proved that the performance of $5^{\text {th }}$-order Gear predictor-corrector scheme is the better compared to other integration methods. This scheme is implemented into the code.

3. The developed DEMMAT code is applied to simulate keel penetration into two-dimensional visco-elastic granular media to investigate the influence of elastic properties of particles. The simulation results show that the increase of elasticity modulus $E$ from lower value $10^{6} \mathrm{~Pa}$ to medium value $10^{8} \mathrm{~Pa}$ provides about 1,9-2,9 times increase of vertical keel's reaction force, while the increase to a higher value $E=10^{11} \mathrm{~Pa}$ gives up to 2,24,3 times reaction increase.

\section{References}

1. Haff, P. K. Discrete mechanics in granular matter. Springer-Verlag, 1993, p. 141-160.

2. Tanaka, K.; Nishida, M.; Kunimochi, T.; Takagi, T. Discrete element simulation and experiment for dynamic response of two-dimensional granular matter to the impact of a spherical projectile. Powder Technology, 124, 2002, p. $160-173$.

3. Owen, D. R. J.; Feng, Y. T. Parallelized finite/discrete element simulation of multi-fracturing solids and discrete systems. Engineering computations, Vol 18, No 3/4, 2001, p. $557-576$.

4. Cundall, P. A.; Strack, O. D. L. A discrete numerical model for granular assemblies. Geotechnique, Vol 29, No 1, 1979, p. 47-65.

5. Kantor, A. L.; Long, L. N.; Micci, M. M. Molecular dynamics simulation of dissotiantion kinetics. In: AIAA Aersospace Sciences Meeting, AIAA Paper 2000-0213, 2000. 
6. Kuwagia, K.; Horio, M. A numerical study on agglomerate formation in a fluidized bed of fine cohesive particles. Chemical Engineering Science, 57, 2002, p. 4737-4744.

7. Cleary, P. W.; Sawley, M. L. DEM modelling of industrial granular flows: 3D case studies and the effect of particle shape on hopper discharge. Applied Mathematical Modeling, 26, 2002, p. 89-111.

8. Li, J.; Mason, D. J. A computational investigation of transient heat transfer in pneumatic transport of granular particles. Powder Technology, 112, 2000, p. 273-282.

9. Tran, T. X.; Dorfman, A.; Rhie, Y. B. Micromechanical modeling of cracking and damage of concrete. In: Computational Modeling of Concrete Structures, Rotterdam, Balkema, 1998, p. 61-69.

10. Džiugys, A.; Peters, B. J. An approach to simulate the motion of spherical and non-spherical fuel particles in combustion chambers. Granular Material, 2001, Vol 3, No 4, p. 231-266.

11. Nardin, A.; Zavarise, G.; Schrefler, B. A. Modelling of cutting tool - soil interaction - part I: contact behaviour. Computational Mechanics, 31, 2003, p. 327-339.

12. Tanaka, H.; Momozu, M.; Oida, A.; Yamazaki, M. Simulation of soil deformation and resistance at bar penetration by the Distinct Element Method. Journal of Terramechanics, 37, 2000, p. 41-56.

13. Yu, H. S.; Mitchell, J. K. Analysis of cone resistance: review of methods. Journal of Geotechnical and Geoenvironmental Engineering, ASCE, 124 (2), 1998, p. 140-149.

14. $\mathrm{PFC}^{3 \mathrm{D}}$ Version 2.0. User's Guide. Itasca Consulting Group, Inc., $78 \mathrm{p}$.

15. Balevičius, R.; Džiugys, A.; Kačianauskas, R. Simulation of penetration in granular media. In: CD-ROM Proceedings of the $15^{\text {th }}$ International Conference on Computer Methods in Mechanics, CMM-2003, 3-6 June, 2003.

16. Kohring, G. A. Computer simulations of granular materials: the effects of mesoscopic forces. J. Phys. I France, Vol 4, 1994, p. 1779-1782.

17. Dury, C. M.; Ristow, G. H. Radial segregation in a twodimensional rotating drum. J. Phys. I France, Vol 7, 1997, p. $737-745$.
18. Kohring, G. A. Dynamical simulations of granular flows on multi-processor computers. In: Computational methods in applied sciences '96, John Wiley \& Sons Ltd., 1996, p. 190-196.

19. Mindlin, R. D.; Deresiewicz, H. Elastic spheres in contact under varying oblique forces. J. Appl. Mech. Trans. ASME, Vol 20, 1953, p. 327-344.

20. Allen, M. P.; Tildesley, D. J. Computer simulation of liquids, Oxford Science Publication, 1990.

21. Thompson, P. A.; Grest, G. S. Granular flow: friction and the dilatancy transition. Physical Review Letters, Vol 67, 1991, p. 1751-1754.

22. Dury, C. M.; Ristow, G. H. Radial segregation in a twodimensional rotating drum. J. Phys. I France, Vol 7, 1997, p. 737-745.

23. Langston, P. A.; Tuzun, U.; Heyes, D. M. Discrete element simulation for granular flow in $2 \mathrm{D}$ and $3 \mathrm{D}$ hoppers: dependence of discharge rate and wall stress on particle interactions. Chemical Engineering Science, Vol 50, No 6, 1995, p. 967-987.

24. Sundaram, S.; Collins, L. R. Numerical considerations in simulating a turbulent suspension of finite-volume particles. Journal of Computational Physics, Vol 124, 1996, p. $337-$ 350.

25. Taguchi, Y-h. New origin of a convective motion: elastically induced convection in granular materials. Physical Review Letters, Vol 69, No 9, 1992, p. 1367-1370.

26. Akiyama, T.; Aoki, K. M.; Iguchi, T.; Nishimoto, K. A fractal properties of vertical vibrated beds of granules. Chemical Engineering Science, Vol 51, No 13, 1996, p. 3551-3553.

27. Kopf, A.; Paul, W.; Dunweg, B. Multiple time step integrators and momentum conservation. Computer Physics Communications, Vol 101, 1997, p. 1-8.

28. Landau, L. D.; Lifshitz, E. M. Theory of elasticity. Volume 7 of Course of Theoretical Physics, Pergamon Press, 1959.

29. Landau, L. D.; Lifshitz, E. M. Mechanics. Volume 1 of Course of Theoretical Physics, Pergamon Press, 1960. 